\title{
LANSCE RADIATION RESISTANT WATER MANIFOLD RETROFIT FOR DC MAGNETS *
}

\author{
Michael J. Borden, James F. O’Hara, Edward M. Perez, Brandon J. Roller, Victor P. Vigil, Lawrence \\ S. Walker, LANL, Los Alamos, New Mexico, 87545, USA.
}

\section{Abstract}

Large maintenance dose burdens have necessitated the development of radiation resistant water manifolds for use on DC magnets in the Proton Storage Ring (PSR), at the Los Alamos Neutron Science Center (LANSCE) accelerator. This paper will describe dose measurements and the mechanical design of radiation-resistant water manifolds used in PSR.

\section{INTRODUCTION}

The Proton Storage Ring at the Los Alamos Neutron Science Center de-ionized water distribution system is hard-plumbed with copper piping throughout the 10-sided ring. Elastomer hose is typically used to distribute water from the main water manifold to the magnets requiring cooling. Electrical isolation and water distribution is also accomplished with elastomer hosing on each magnet. The PSR equipment has recently experienced failure of the elastomer hoses at an unacceptable rate in the highest radio-activated regions.

\section{DOSE MEASUREMENTS}

Radio-chromic film has been distributed throughout the PSR to measure total dose exposure for a typical yearly run cycle of 6-8 months. A dosimeter film aluminum foil pack may be seen in Figure 1 of a PSR bump magnet.

The two high-radiation areas addressed recently are the PSR bump magnet system and the large dc C-magnet immediately following beam injection and stripping. Figures 1 and 2 show the magnets prior to upgrades to more radiation resistant materials. Table 1 summarizes the total dose these manifolds receive during a 1-year beam cycle.

\section{Measurement Technique}

Radiochromic film is a nylon matrix that is impregnated with leuco-dye Hexa(hydroxyethyl) paraosaniline nitrile. The dye changes color when subjected to radiation damage and turns cobalt blue as the material is subject to greater and greater radiation doses. The color changes are linearly related to the dose received. Three ranges of radiochromic film are used at the LANSCE facility. The three different films are made from different thicknesses of the same material. The thin film has the least sensitivity and thus the largest dose range $(50,000 \mathrm{rad}-$ $20 \mathrm{Mrad})$. The next film is three times as thick and has a medium range $(1,000 \mathrm{rad}$ to $100,000 \mathrm{rad})$, and the thickest film has a low range (150 rad to 5,000 rad) [1].

\footnotetext{
* Work supported by the United States Department of Energy, National Nuclear Security Agency, under contract DE-AC5206NA25396.
}

06 Instrumentation, Controls, Feedback \& Operational Aspects
Three films are loaded into sealed aluminum packets and exposed for the duration of the run cycle. They are then collected and read out on a colorimeter at 600 and 510 nanometers.

A set of films have been exposed to a known standard dose. These films are then used as the means of calibrating the system.

\section{Worker Considerations}

When workers must make an entry to repair a failed hose, they are exposed to radiation fields up to 500 millirem per hour at 30 centimeters. One hose repair can easily accumulate 100 millirem of exposure for an individual. Each hose failure also results in 2-4 hours of downtime to make repairs and recover the de-ionized water system.

Table 1: PSR Dose Measurements

\begin{tabular}{|l|l|l|}
\hline No. & PSR equipment description & $\begin{array}{l}\text { Total dose for 8 } \\
\text { month run, 2006 }\end{array}$ \\
\hline 332 & Upstream wire scanner & 1 e 6 rads \\
\hline 333 & $\begin{array}{l}\text { Water hose at upstream } \\
\text { quadrupole }\end{array}$ & 1.3 e 6 rads \\
\hline 334 & Water hose at C-magnet & 9.5 e 5 rads \\
\hline 335 & Power cables at C-Magnet & 1 e 6 rads \\
\hline 336 & $\begin{array}{l}\text { Floor TV camera at } \\
\text { injection point }\end{array}$ & 1.2 e 5 rads \\
\hline
\end{tabular}

\section{MANIFOLD UPGRADES}

Two approaches were used to upgrade these two magnet systems to radiation-resistant materials. Each approach will be discussed separately.

\section{PSR Bump Magnets}

As can be seen in figure 1, the elastomer water distribution hoses on the top of the bump magnets are easily accessed. The hoses do have tight radii with large induced stresses.

In this case the decision was made to completely eliminate the elastomer hose. 5 millimeter diameter copper tubing was soldered to ceramic electrical isolating assemblies. The soldered assemblies were then terminated with 5 millimeter brass Swagloc ${ }^{\mathrm{TM}}$ nuts and compression ferrules to allow attachment to the distribution manifold and magnet coils. The larger 10 millimeter diameter elastomer supply and return hoses were also replaced with 10 millimeter copper tubing. 10 millimeter brass Swagloc ${ }^{\mathrm{TM}}$ nuts and compression ferrules were used to connect each end to the magnet manifold and main water distribution manifold. A completed magnet can be seen in figure 2. This repair was completed in the spring of 2006 . 
The bump magnet system has operated without water system failures since that time.

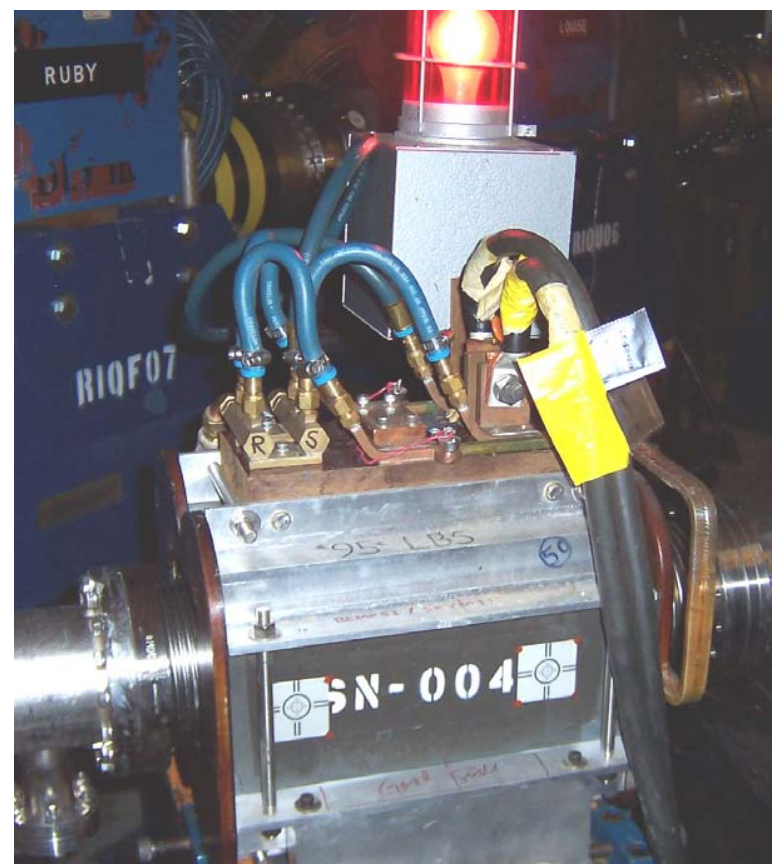

Figure 1: Hosed PSR bump magnet with dose packet.

\section{PSR C-Magnets}

The first C-magnet in PSR receives multiple scattering losses from beam interaction with the upstream carbon stripper foil. Several hose failures have occurred over the past two run cycles. Figures 3 and 4 show the complexity of the upper and lower coil water distribution system.

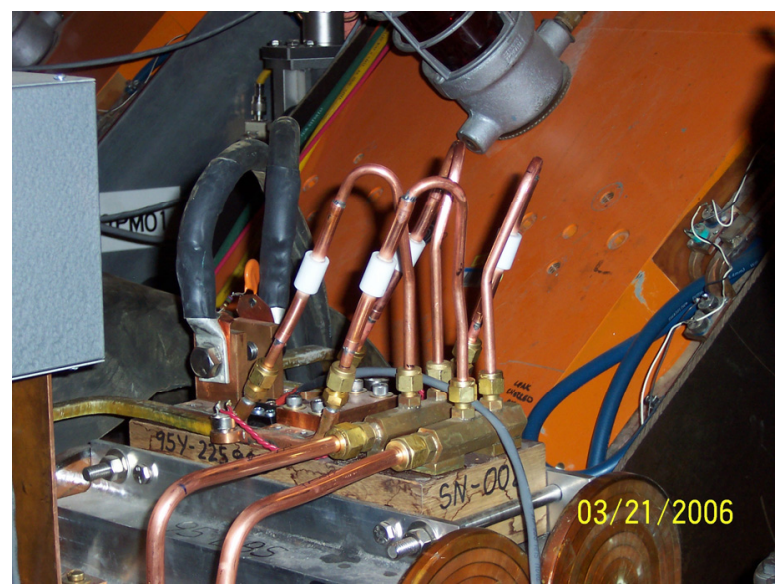

Figure 2: Radiation-resistant connections on bump magnet.

The connections to the magnet coils and distribution manifold have as little as 3 millimeters of clearance and are partially obscured by the main coil power connections. The back inside row of the coil connections cannot be accessed without removing part of the front row. Disturbing other connections frequently resulted in creating new water leaks and more beam downtime. The number and complexity of the C-magnet water distribution circuits necessitated a different approach to make the system radiation-resistant.

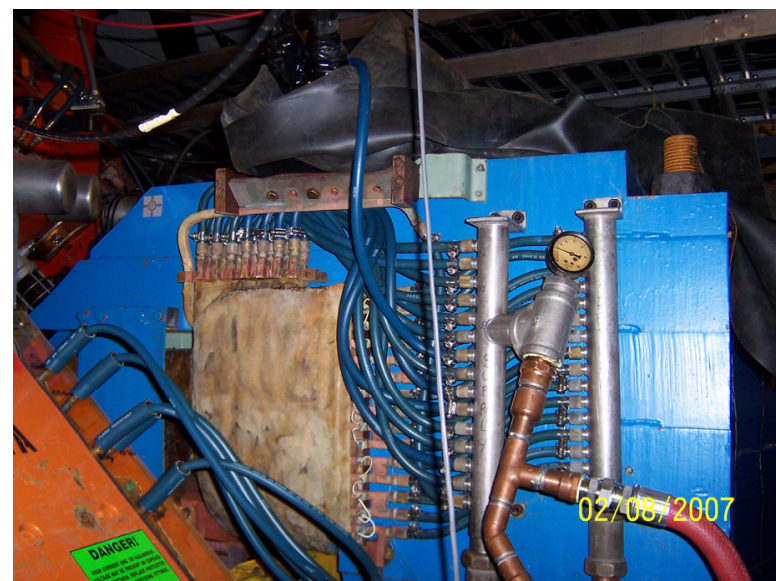

Figure 3: Upper-hosed PSR C-magnet coil.

In this case, as seen in figure 5 the transition back to electrically isolating, elastomer hoses has been moved nearly a meter away from the high-radiation area. The extension was accomplished by brazing Lawrence Radiation Laboratory (LRL) fittings to 10 millimeter diameter copper tubing. The copper tubing is insulated with black woven fiberglass sleeves. The insulated copper extensions are supported and routed with phenolic blocks. The upper and lower levels of the extensions are separated to allow easy hose or fitting maintenance.

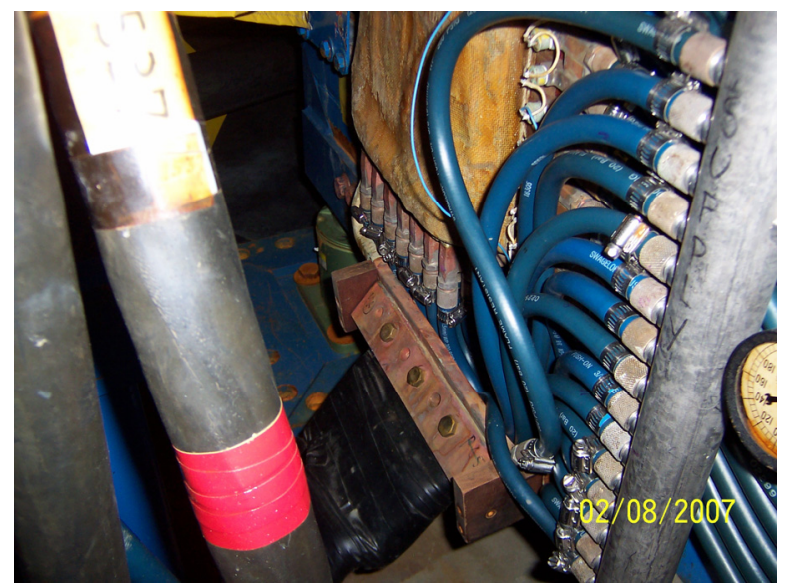

Figure 4: Lower-hosed PSR C-magnet coil.

The 10 millimeter diameter elastomer hose is connected with LRL and brass Swagloc TM nuts and compression ferrules to the copper tubing extensions and water manifolds, respectively. The LRL o-rings seals have been replaced with radiation-resistant Kalrez ${ }^{\mathrm{TM}}$ o-rings. The magnet water manifolds are mounted on the back of the magnet iron yoke, which provides significant radiation shielding to workers. All protective thermal switch wiring has been replaced with radiation-resistant Kapton ${ }^{\mathrm{TM}}$ insulated wire. The entire assembly was finally covered with quick release phenolic and polycarbonate sheet to prevent inadvertent access to electrical connections, but allows ease of maintenance.

T18 Radiation Monitoring and Safety 


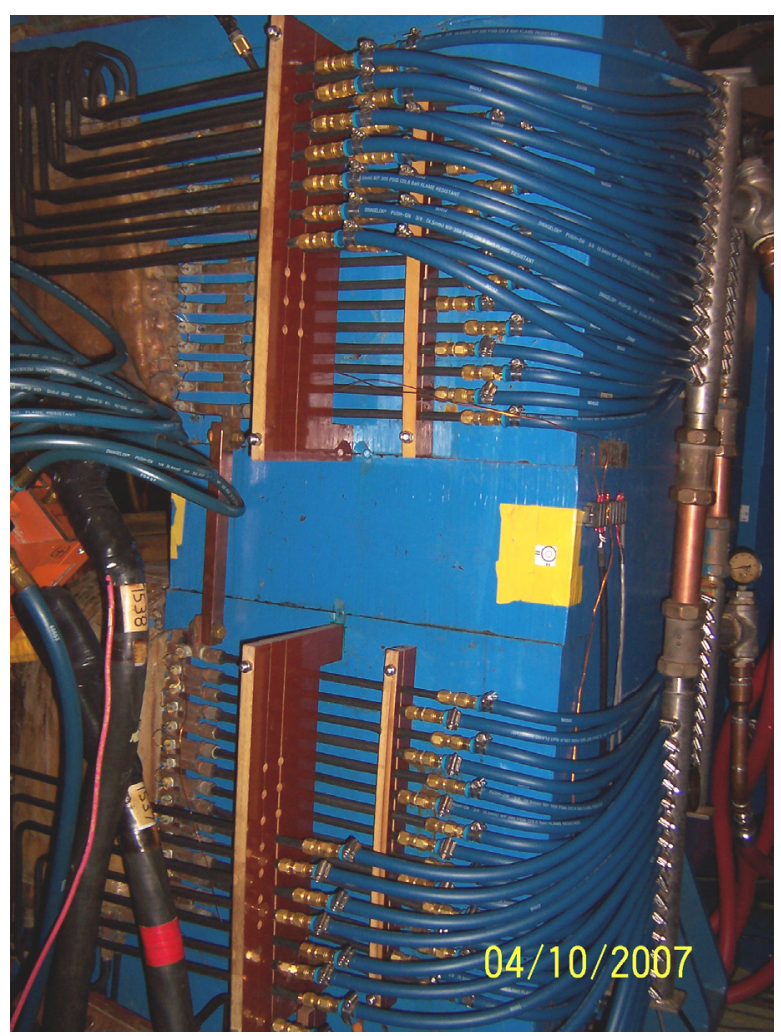

Figure 5: Radiation-resistant PSR C-magnet coil manifold.

\section{REFERENCES}

[1] Azam Niroomand-Rad, etal, Radiochromic Film Dosimetry, Radiation Therapy Committee Task Group No. 55, AAPM Report No. 63, reprinted from MEDICAL PHYSICS, Vol. 25, Issue 11, November 1998. 